\title{
Correction to: Neuronopathic Gaucher disease presenting with microcytic hypochromic anemia
}

\author{
Eun Ah Kim ${ }^{1}$. Young Tae Lim ${ }^{1}$. Jeong Ok Hah ${ }^{2}$. Young Bae Sohn ${ }^{3}$. Yu Kyung Kim ${ }^{4}$. Joon Hyuk Choi ${ }^{5}$. \\ Sae Yoon Kim ${ }^{1}$. Kyung Mi Jang ${ }^{1}$. JiYoung Ahn ${ }^{1}$. Jae Min Lee ${ }^{1}$ (i)
}

Published online: 10 January 2019

(C) Japanese Society of Hematology 2019

\section{Correction to: International Journal of Hematology https://doi.org/10.1007/s12185-018-2559-3}

In the original publication of this article, Fig. 2 was published incorrectly. The correct Fig. 2 is given in the following page.

Publisher's Note Springer Nature remains neutral with regard to jurisdictional claims in published maps and institutional affiliations.

The original article can be found online at https://doi.org/10.1007/ s12185-018-2559-3.

\section{Jae Min Lee}

mopic@ hanmail.net

1 Department of Pediatrics, Yeungnam University College of Medicine, Daegu, Republic of Korea

2 Department of Pediatrics, Daegu Fatima Hospital, Daegu, Republic of Korea

3 Department of Medical Genetics, Ajou University Hospital, Ajou University School of Medicine, Suwon, Republic of Korea

4 Department of Clinical Pathology, School of Medicine, Kyungpook National University, Daegu, Republic of Korea

5 Department of Pathology, Yeungnam University College of Medicine, Daegu, Republic of Korea 
$\multimap \mathrm{WBC} \rightarrow \mathrm{Hb} \rightarrow$ Platelet

16

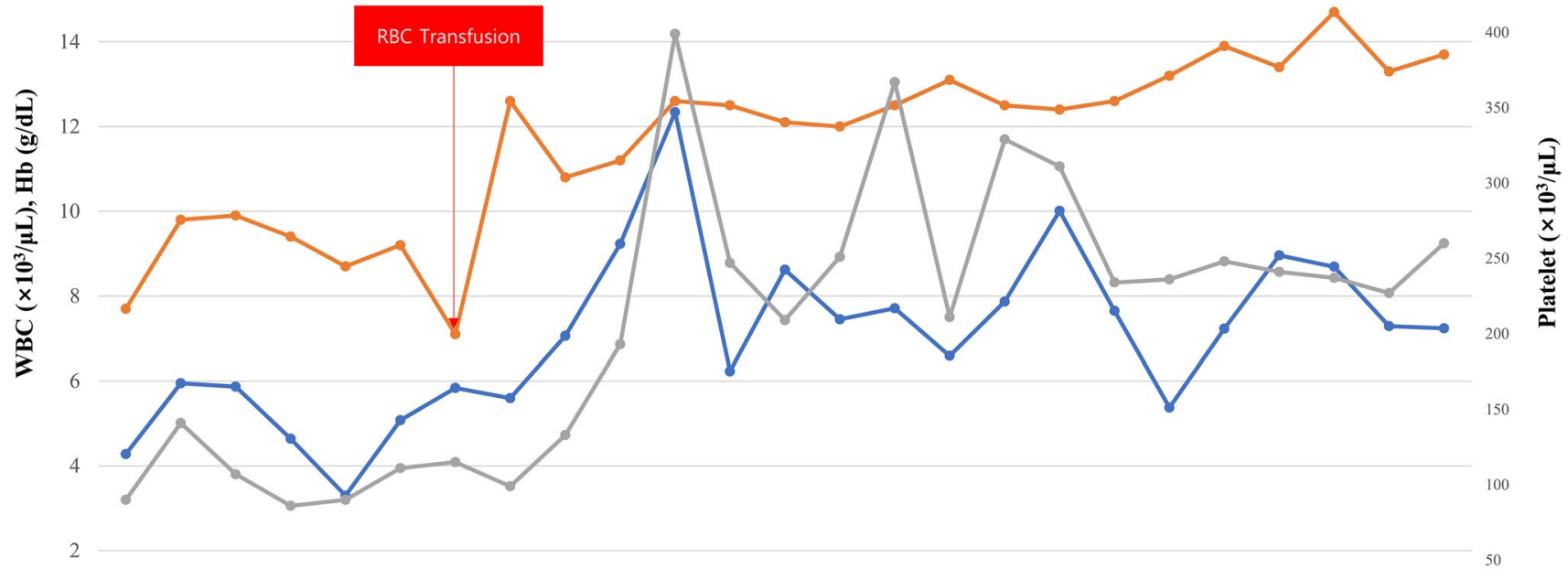

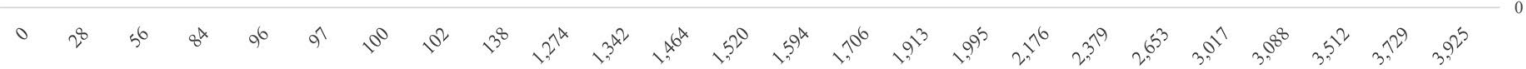

Time after first visit (days)

Iron

Imiglucerase

Fig. 2 Hematologic profile change during ERT, RBC TF, and red blood cell transfusion 